\title{
The Barotropic Instability of the Oceanic Jet Currents
}

\author{
Alexander Alekseyevich Solovyev ${ }^{1}$, Dmitry Alexandrovich Solovyev ${ }^{2,}$, \\ ${ }^{1}$ Department of Geography, M. V. Lomonosov Moscow State University, Moscow, Russia \\ ${ }^{2}$ Sea-Air Interaction and Climate Laboratory, P. P. Shirshov Institute of Oceanology RAS, Moscow, Russia
}

\section{Email address:}

geosolmgu@mail.ru (A. A. Solovyev), solovev@ocean.ru (D. A. Solovyev)

${ }^{*}$ Corresponding author

\section{To cite this article:}

Alexander Alekseyevich Solovyev, Dmitry Alexandrovich Solovyev. The Barotropic Instability of the Oceanic Jet Currents. Hydrology. Vol. 5, No. 5, 2017, pp. 77-81. doi: 10.11648/j.hyd.20170505.13

Received: February 21, 2017; Accepted: March 28, 2017; Published: November 1, 2017

\begin{abstract}
In this article we present the results of the laboratory experiments with shift currents in the homogenous shallow water generated by differentially rotating elements of the vessel bottom: central axisymmetric disk and outer coupled rings. Based on the experimental results, we explain the generation of vortex rings due to the development of barothropic or shift instability of currents. We show that the surface of the differentially rotating liquid in the horizontal rate shift zone creates the conditions for generating chains of vortices rotating clockwise. When changing the values of rate shift and the rotation rate of the whole system, the formation of modes of instability of vortex structures is observed, the conditions for which were quantified on the laboratory current stability diagram in Rossby and Ekman numbers. To interpret the experimental data we considered the solutions of the equations for quasi-two-dimensional geophysical currents in the form of elementary waves of the current function disturbance. We estimated the parameters of the perturbations development at the meanders of the different parts of Gulf Stream using the calculation of the increment of experimental curves for neutral stability. The evaluation results provide a basis for the development of realistic approaches to understanding the processes of generation and evolution of synoptic vortices in the meanders of intense oceanic jet currents.
\end{abstract}

Keywords: Jet Currents in the Ocean, Barotropic Instability, Modeling of Currents, Laboratory Experiment

\section{Introduction}

Due to the development of the general atmosphere and ocean circulation theory the particular attention is paid to the studies of the impact of both baroclinic and barotropic processes caused by (respectively) vertical and horizontal rate shifts, on the development of the instability of largescale oceanic currents $[1,2]$. The research of meandering processes of large oceanic currents leading to the formation of ring vortices from large meanders was carried out in some works $[3-8]$. We considered the problem of specifying the mechanism of the formation of rings due to the baroclinic instability of oceanic jet currents, the development of which is possible only in the rotating liquid stratified by density [6]. The typical size of vortices associated with the local baroclinic Rossby radius of deformation exceed it in several times [7]. Jet currents belong to the class of motions in geological environments where horizontal shifts can have a significant impact on the formation of the barotropic instability of hydrodynamic processes in the ocean. First of all we should consider the vortex rings in the Gulf Stream type systems of jet currents [8]. The shift instability occurring during the horizontal rate steps may lead to the formation of vortices, the radius of which is typically lesser than Rossby radius of deformation [7]. These vortices are observed on the outer periphery of Gulf Stream jet. Some contribution to the establishment of the physical mechanisms of the dominant impact on the dynamics of ocean currents is made by the laboratory hydrodynamic experiments $[3-9$, 10]. Researching the models with rotating liquids in the wide range of wave numbers and frequencies provides the dynamic similitude with the ocean-scale processes [3, 5]. The laboratory observations of the analogues of oceanic processes, like subscale and mesoscale vortices, and energetic estimates of the baroclinic instability of various scale vortices revealed both forward and back energy transfer cascades [11]. Of equal interest for understanding the dynamics of the development of large-scale oceanic 
processes is the problem of the stability of the system of two opposed jet currents in the rotating layer of shallow water and its solution based on the experimental research of jet currents with horizontal rate shift in the laboratory model, where the barothropic instability is presented in its pure form. Our subject was the experimental research of jet currents with horizontal rate shift of the laboratory ring model, in which the barotropic instability presents in the pure form. In this work we consider the impact of the barotropic effects on the meandering of the oceanic jet currents and ring formation based on the laboratory experiments with differentially rotating shallow liquid.

\section{Materials and Experimental Setup}

To simulate the barotropic instability of jet currents we used cylindrical vessel in which the jet current was generated by the system of rings at the bottom, rotating relative to each other and to the vessel [12]. The horizontal rate shift was created by the independent rotation of the disk and two rings placed on the flat bottom of the vessel (see Fig. 1).

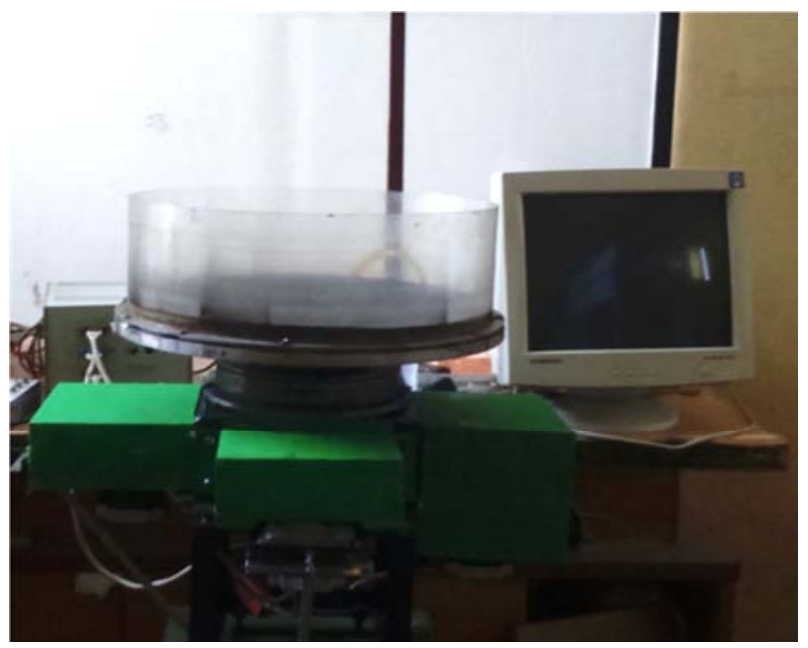

Figure 1. General view of the experimental setup.

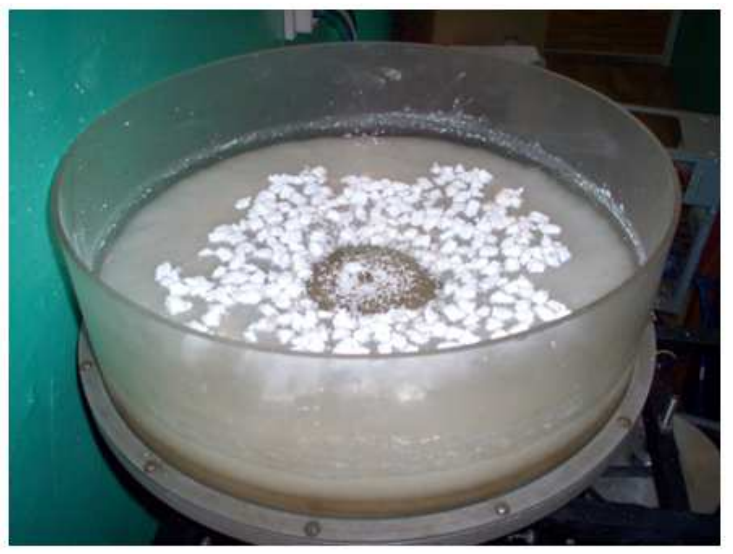

a)
Disk and rings were placed precisely at the same level. The axes of the disk and rings were placed outside the vessel so that any part of the vessel bottom was available for the rotating water. Vessel height was $25 \mathrm{~cm}$, diameter of the disk $-20 \mathrm{~cm}$, central ring width $-10 \mathrm{~cm}$, outer ring width $-3 \mathrm{~cm}$. The experimental setup allowed combining different rotation modes of the bottom segments. During the experiments with the barotropic instability of jet currents the disk was rotating with the rate $\omega_{1}$ which was different from the rates $\omega_{2}$ and $\omega_{3}$ of the central and outer rings, respectively. The angular rates of the rings were equal. Disk and coupled rings rotated in the same counterclockwise direction. We observed the condition of the small thickness of the undisturbed water $\mathrm{H}_{\mathrm{o}}=0.025 \mathrm{~m}$ with respect to the typical horizontal scale of the current. The rotating rate $\omega_{0}$ of the structures generated at the rate shifts remained lesser than the rotating rate $\Omega$ of the whole system. The ratio of the Obukhov-Rossby radius $\mathrm{r}^{*}=\sqrt{\frac{\mathrm{gH}_{\mathrm{o}}}{2 \Omega}}$ to the size of the vortex structures $a$ was close to $1\left(\mathrm{r}^{*} / \mathrm{a} \approx 1\right)$. An important feature of these experiments was the condition $\Delta<\mathrm{a}$, where $\Delta=\mathrm{H}_{\mathrm{o}} \mathrm{E}^{1 / 4}$ is the thickness of the Proudman-

Stewartson bottom layer; $\mathrm{E}=\frac{v}{\Omega \mathrm{H}_{\mathrm{o}}^{2}}-$ Ekman number; $v-$ kinematic viscosity of the water. The effect $\beta$ was provided by the dependency of the effective thickness $\mathrm{H}$ of the layer of rotating water in the radial direction. The profile of the current in the experiments corresponded to the stepwise rate shift that was created on the end surface of the vessel at the border where the disk contacts the ring. By setting different rotating rates for the center and periphery the ctationary form and amplitude of the shift was defined. To characterize currents we used, besides the Ekman number, the Rossby shift number $R o=\frac{\Delta \omega}{2 \Omega}$, in which the rate shift $\Delta \omega$ was defined by the difference between the rotating rates of the disk and the coupled rings.

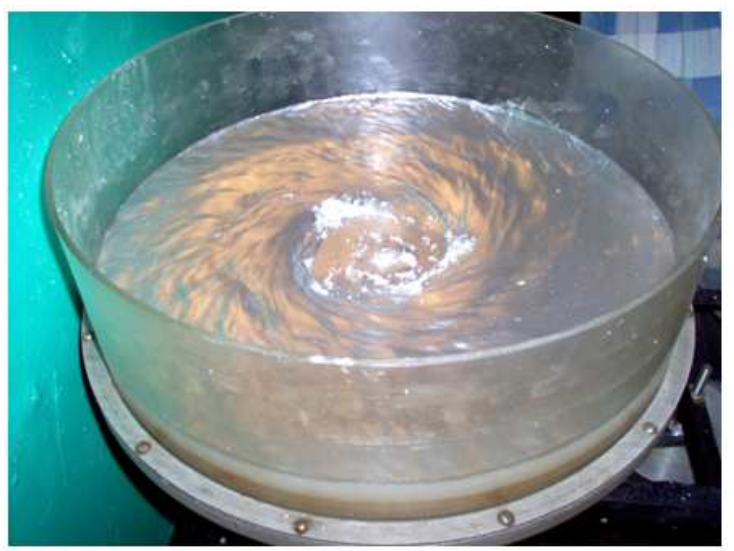

b)

Figure 2. Photos of the current structure under the shift rotation of the shallow water. Left (a) is three-mode wave contour bounding the wave system on the edge of the rate shift zone (right (b)). 
We found that various stable current line patterns are formed on the liquid surface (Fig. 2). In the central part they were forming various figures with different symmetry indexes from the visualizing particles. At the periphery zone of the rotating shallow water the crests and troughs of the waves were forming [13]. Their axes were oriented along the radius or at some angle to it. The wave system was a chain of vortices with clockwise rotation. Their number was equal to the number of sides of the polygonal structures rotating around the vessel axis. When the values of the rate shift $\Delta \omega$ and the rotating rate of the whole system $\Omega$ was changed synchronously and discretely, we observed two different scenarios of the instability development. Increasing the shift intensity resulted in reducing the number of vortices; increasing the whole rotating rate and, respectively, decreasing the width of the shift zone resulted in the increasing the number of vortices while keeping the nondimensional wave number. According to the results of the measurements we calculated Ro and $\mathrm{E}$ numbers for supercritical conditions and constructed a laboratory current stability graph (Fig. 3) [8].

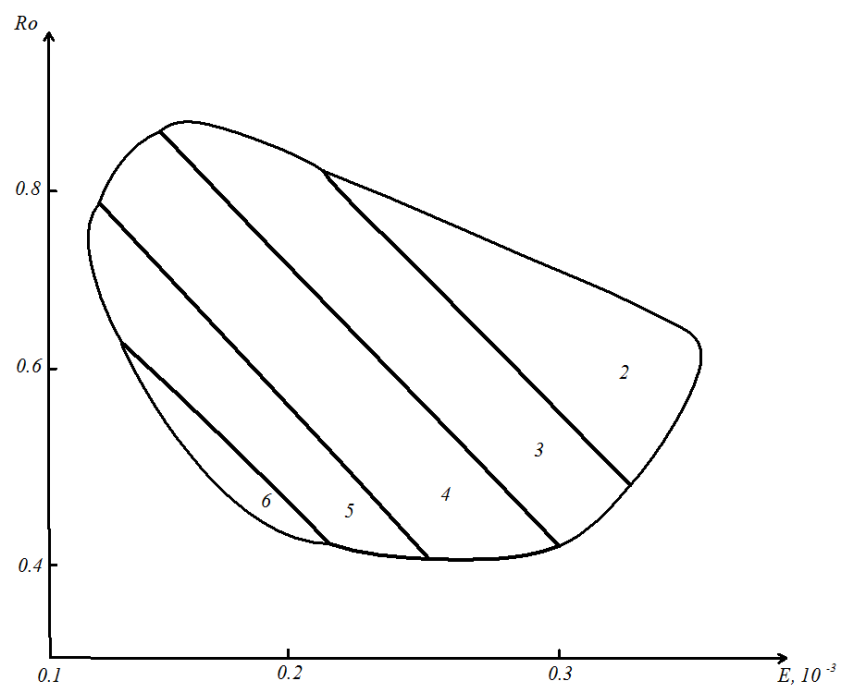

Figure 3. Diagram of the stability of shift perturbations of differentially rotating shallow water at $H=0.025 \mathrm{~m}$. Numbers show the wave numbers of the asymmetric rotation modes.

To define quantitative characteristics of the stability threshold for such conditions with different nondimensional wave number values we used inverse power dependence between Rossby number and Ekman number which characterizes the experimental situation rather adequately.

\section{Results and Discussion}

Analysis of the results of laboratory experiments was carried out only relating to Gulf Stream. To describe the laboratory experiments, just like for the full-scale currents, we considered the solutions of the equation for quasi-twodimensional geophysical currents in the form of elementary wave in the form of the current function perturbation:

$$
\Psi(\mathrm{x}, \mathrm{y}, \mathrm{z})=\phi(\mathrm{y}, \mathrm{z}) \mathrm{e}^{\mathrm{ik}(\mathrm{x}-\mathrm{ct})}
$$

Where $\phi(\mathrm{y}, \mathrm{z})$ is wave amplitude; $\mathrm{k}$ - wave number; $\mathrm{c}=\mathrm{c}_{\mathrm{r}}+i \mathrm{c}_{\mathrm{i}}-$ complex phase velocity. Value $\gamma_{\mathrm{i}}=\mathrm{c}_{\mathrm{i}} \mathrm{k}-$ is the increment coefficient for the imaginary part of the complex frequency which characterizes the amount of the exponential change of the wave amplitude during its propagation.

To make a transition from the laboratory experiments to full-scale currents we propose the following calculation scheme. The nondimentional number $\alpha$ in the model is defined by the equation [10]: $\alpha=\frac{k}{k_{0}}$, where $k=\frac{2 \pi}{r_{2}-r_{1}}$ is radial wave number; $k_{0}=\frac{2}{r_{2}+r_{1}}-$ azimuthal wave number; $\mathrm{n}$ - mode number of radial modes, $\mathrm{m}$ - mode number of azimuthal modes. Take mode number of radial modes equal to $\mathrm{n}=1$. In our case, with radiuses of the rings $r_{1}=0.23 \mathrm{~m}$, $r_{2}=0.1 \mathrm{~m}$, the wave number equals $\alpha=0.125 \mathrm{~m}$. Now proceed from the horizontal rates and horizontal rate shifts to the rate value $u_{m}$ of the main jet current using the following equation:

$$
\mathrm{u}_{\mathrm{m}}=\frac{\left(\omega_{1}-\omega_{2}\right) \mathrm{r}_{1}}{2}
$$

Curves $\mathrm{u}_{\mathrm{m}}=\mathrm{f}(\alpha)$ aren't illustrative enough for practical evaluation of the perturbation increase degree. More useful to compare laboratory and full-scale data are stability diagrams in the form of family of curves $u=f(L)$, where the average flow rate across the current is multiplied by the transition scale factor $\mathrm{u}=50 \cdot \mathrm{u}_{\mathrm{m}}$. The dimensional length of the perturbation wave L considering the laboratory and full-scale scales equates the following:

$$
L=\frac{100}{\alpha}(\mathrm{km})
$$

The revaluation of the neutral stability curves obtained in the laboratory tests to the full-scale ones with isolines in the form of increase factors $\gamma_{t}$ was performed using the following equation:

$$
\gamma_{l}=\frac{v}{H^{2}}
$$

To evaluate the values of horizontal turbulent viscosity coefficient we choose the value equal to $10^{8} \frac{\mathrm{cm}^{2}}{\mathrm{sec}}$. During laboratory tests we took the value of this coefficient equal to $10^{-2} \frac{\mathrm{cm}^{2}}{\mathrm{sec}}$. Considering the ratio between laboratory and fullscale scales is $10^{-7}$, using Eq. 3 calculate the value: 


$$
\gamma_{l}^{\text {full }}=\frac{10^{-4} v_{l a b}}{H_{l a b}^{2}}=\frac{10^{-6}}{H_{l a b}^{2}}
$$

From the isolines family $\gamma_{l}=$ const (see Fig. 4) characterizing the instability development in the jet current we can see that the value of the dominating wavelength doesn't depend on the horizontal rate shift.

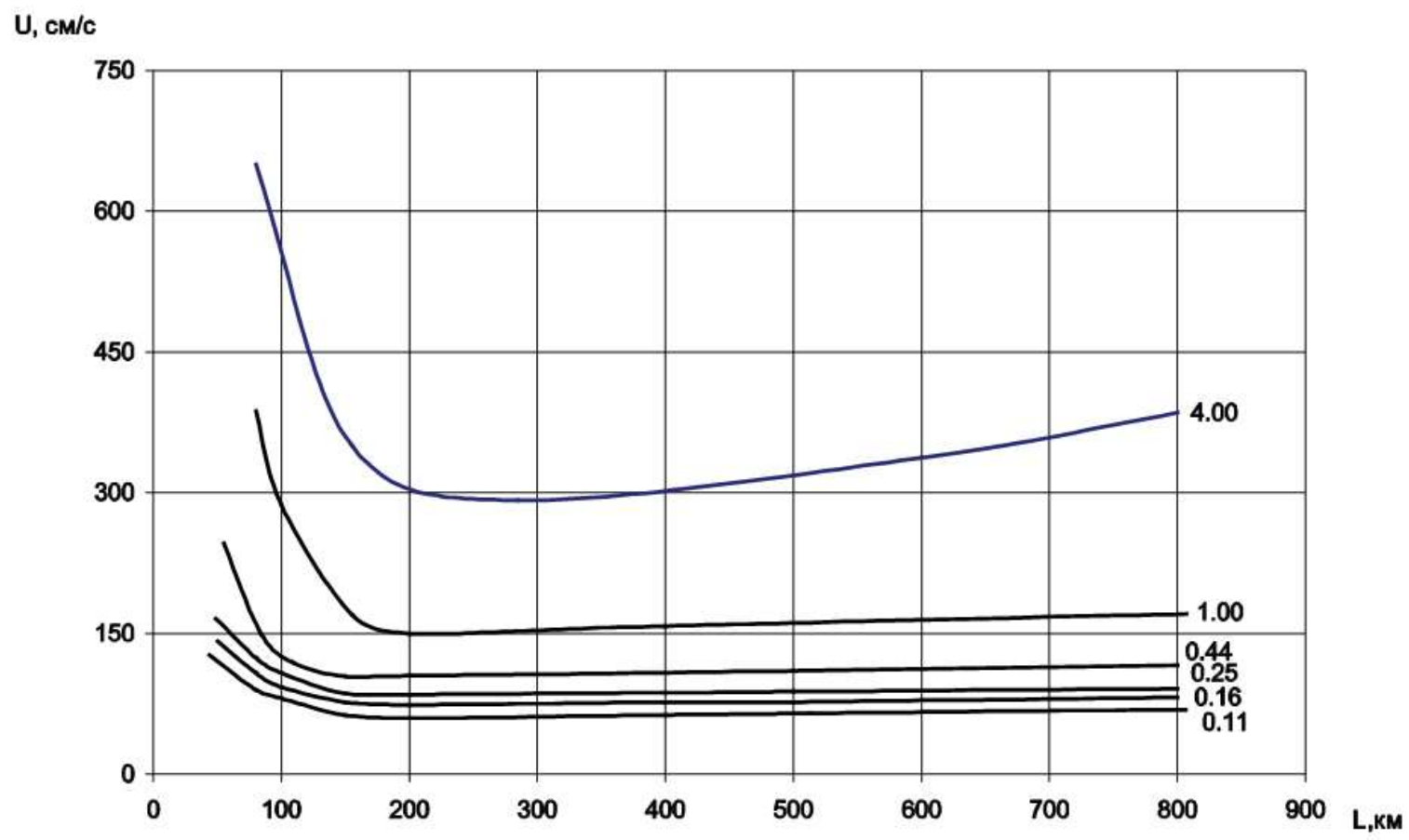

Figure 4. Neutral curves shown as isolines of increase factors $\gamma_{l}$ (their values are multiplied by the numerical factor $10^{6}$ ).

Now proceed to the evaluation of the perturbation development parameters caused by the meanders in the different parts of Gulf Stream. According to the definition of the increase factor, the perturbation amplitude should increase twice at the time $\mathrm{T}$ equal to

$$
T=\ln \frac{2}{\gamma_{l}}
$$

where. $\left[\gamma_{t}\right]=c^{-1}$ Consider as an example two cross-sections of Gulf Stream with coordinates (Tab. 1): $68^{\circ} 28^{\prime} w, 38^{\circ} 50^{\prime} n$ and $65^{\circ} 06^{\prime} w, 38^{\circ} 19^{\prime} n$ [13]. Value $\gamma_{l}$ was defined using the measured values of $\mathrm{u}, \mathrm{L}$.

Table 1. Parameters of barotropic instability in meandering of the Gulf Stream.

\begin{tabular}{lll}
\hline Parameters & Cross-sections 1 & Cross-sections 2 \\
\hline $\mathrm{L}, \mathrm{km}$ & 160 & 200 \\
$\mathrm{~m}$ & 5 & 4 \\
$\gamma_{l}, \mathrm{sec}^{-1}$ & $1.610^{-2}$ & $2.910^{-4}$ \\
$\mathrm{~T}$, day & 3 & 7 \\
\hline
\end{tabular}

This result satisfactorily conformed with the observed data in [14], where it was noted that the meander amplitude in Gulf Stream increases twice during about 10 days. The calculations and observations were in compliance also for the wavelength as for wavelength [5]. The scale spectrum of its data has its peak near $200 \mathrm{~km}$. Some overestimated values of the factor $\gamma_{l}$, and hence low values of $\mathrm{T}$ are due to the fact that in the calculations the turbulent viscosity coefficient was taken with excess. This somewhat increased the value of the stabilizing factor.

\section{Conclusion}

The results of experiments with the resolution of shallow water give the following conclusions. Barotropic instability of currents is a source of generation of vortex rings from meander jet currents in the ocean with dimensions close to the Obukhov-Rossby deformation radius. The occurrence of a different number of vortex rings is possible under conditions of shear instability of current, because of a change in the shear rate and the velocity of the jet current. The estimates show the parameters of the development of barotropic perturbations caused by the meandering of various sections of the Gulf Stream. The evaluation results presented here provide a basis for a more realistic perspective on the features of the formation of meanders in ocean currents.

\section{Acknowledgements}

The research was funded by the grant of the Russian Scientific Fund (project No. 14-50-00095). 


\section{References}

[1] G. Korotaev, "Structure, Dynamics And Energetics of the Synoptic Instability of the Ocean," Sevastopol, 1984.

[2] A. Monin and G. Zhikharev, "Oceanic Vortices," UFN, vol. 5, no. 160 , pp. $1-47,1990$.

[3] V. V. Alekseev, S. V. Kisileva, and S. S. Lappo, Laboratory models of the physical processes in the atmosphere and ocean. Moscow: Nauka, 2005.

[4] Ø. Thiem, J. Berntsen, and B. Gjevik, "Development of eddies in an idealised shelf slope area due to an along slope barotropic jet," Cont. Shelf Res., vol. 26, no. 12, pp. 14811495, 2006.

[5] K. Wyrtki, L. Magaard, and J. Hager, "Eddy energy in the oceans," J. Geophys. Res., vol. 81, no. 15, pp. 2641-2646, 1976.

[6] K. N. Fedorov, The physical nature and structure of oceanic fronts. Leningrad: Gidrometeoizdat, 1983.

[7] V. M. Kamenkovich, M. N. Koshlyakov, and A. S. Monin,

Synoptic eddies in the ocean. Leningrad: Gidrometeoizdat, 1982.

[8] G. Stommel, Gulf Stream: Physical And Dynamical Description. Moscow: Inostrannaya Literatura, 1963.

[9] F. Dolzhanskiy, V. Krymov, and D. Manin, "Stability And Vortice Structures of the Quazi-two-dimensional Shift Currents," UFN, vol. 7, no. 160, pp. 1-47, 1990.

[10] H. Greenspen, Theory of Rotating Fluids. Leningrad: Gidrometeoizdat, 1973.

[11] Y. Zhang and Y. D. Afanasyev, "Baroclinic turbulence on the polar $\beta$-plane in the rotating tank: Down to submesoscale," Ocean Model., vol. 107, pp. 151-160, 2016.

[12] V. M. Lushin and C. C. Lappo, "Geohydraulic model," A. c. 647572, 1979.

[13] D. Soloviev, A. Soloviev, and K. Pavlovskiy, "Diagram of the Wave Stability in the Differentially Rotating Shallow Water," in Currents And Structures in Fluids. Proceedings of the International Conference., 2005, pp. 42-44.

[14] T. Lee and P. Cornillon, "Propagation of Gulf Stream meanders," J. Phys. Ocean., vol. 26, pp. 225-241, 1996. 